\title{
(2) OPEN ACCESS \\ Changes in the behavioural determinants of health during the COVID-19 pandemic: gender, socioeconomic and ethnic inequalities in five British cohort studies
}

\author{
David Bann @ (, ${ }^{1}$ Aase Villadsen, ${ }^{1}$ Jane Maddock, ${ }^{2}$ Alun Hughes, ${ }^{2}$ George B. Ploubidis, \\ Richard Silverwood, ${ }^{1}$ Praveetha Patalay (1) ${ }^{1,2}$
}

\begin{abstract}
- Additional supplemental material is published online only. To view, please visit the journal online (http://dx. doi.org/10.1136/jech-2020215664)
\end{abstract}

${ }^{1}$ Centre for Longitudinal Studies, Social Research Institute, University College London, London, UK

${ }^{2}$ MRC Unit for Lifelong Health and Ageing, Research Department of Population Science and Experimental Medicine, University College London, London, UK Dr David Bann, Social Research Institute, University College London, London, London, UK; david.bann@ucl.ac.uk

$\mathrm{RS}$ and PP contributed equally.

Received 24 September 2020 Accepted 13 May 2021 Published Online First 26 May 2021
Correspondence to

\section{ABSTRACT \\ Background The COVID-19 pandemic is expected to have far-reaching consequences on population health. We investigated whether these consequences included changes in health-impacting behaviours which are important drivers of health inequalities.}

Methods Using data from five representative British cohorts (born 2000-2002, 1989-1990, 1970, 1958 and 1946), we investigated sleep, physical activity (exercise), diet and alcohol intake ( $\mathrm{N}=14297)$. We investigated change in each behaviour (pre/during the May 2020 lockdown), and differences by age/ cohort, gender, ethnicity and socioeconomic position (childhood social class, education attainment and adult financial difficulties). Logistic regression models were used, accounting for study design and non-response weights, and meta-analysis used to pool and test cohort differences in association.

Results Change occurred in both directions - shifts from the middle part of the distribution to both declines and increases in sleep, exercise and alcohol use. Older cohorts were less likely to report changes in behaviours while the youngest reported more frequent increases in sleep, exercise, and fruit and vegetable intake, yet lower alcohol consumption. Widening inequalities in sleep during lockdown were more frequent among women, socioeconomically disadvantaged groups and ethnic minorities. For other outcomes, inequalities were largely unchanged, yet ethnic minorities were at higher risk of undertaking less exercise and consuming lower amounts of fruit and vegetables.

Conclusions Our findings provide new evidence on the multiple changes to behavioural outcomes linked to lockdown, and the differential impacts across generation, gender, socioeconomic circumstances across life, and ethnicity. Lockdown appeared to widen some (but not all) forms of health inequality.

\section{INTRODUCTION}

The COVID-19 pandemic is expected to have (c) Author(s) (or their employer(s)) 2021. Re-use permitted under CC BY. Published by BMJ.

To cite: Bann D, Villadsen A, Maddock J, et al. J Epidemiol Community Health

2021:75:1136-1142. far-reaching consequences on population health, particularly in already disadvantaged groups. ${ }^{1} 2$ Aside from direct effects of COVID-19 infection, detrimental changes may include effects on physical and mental health due to associated changes to health-impacting behaviours. Change in such behaviours may be anticipated due to the effects of social distancing, both mandatory and voluntary, and change in factors which may affect such behaviours-such as employment, financial circumstances and mental distress. ${ }^{34}$ The behaviours investigated here include physical activity, diet, alcohol and sleep ${ }^{5}$-likely key contributors to existing health inequalities ${ }^{6}$ and indirectly implicated in inequalities arising due to COVID-19 given their link with outcomes such as obesity and diabetes. ${ }^{7}$

While empirical evidence of the impact of COVID-19 on such behaviours is emerging, ${ }^{8-26}$ it is currently difficult to interpret for multiple reasons. First, generalising from one study location and/or period of data collection to another is complicated by the vastly different societal responses to COVID-19 which could plausibly impact on such behaviours, such as restrictions to movement, access to restaurants/pubs and access to support services to reduce substance use. This is compounded by many studies investigating only one health behaviour in isolation. Further, assessment of change in any given outcome is notoriously methodologically challenging. ${ }^{27}$ Some studies have questionnaire instruments which appear to focus only on the negative consequences of COVID $-19,{ }^{8}$ thus curtailing an assessment of both the possible positive and negative effects on health behaviours.

The consequences of COVID-19 lockdown on behavioural outcomes may differ by factors such as age, gender, socioeconomic position (SEP) and ethnicity-thus potentially widening already existing health inequalities. For instance, younger generations (eg, age 18-30 years) are particularly affected by cessation or disruption of education, loss of employment and income ${ }^{3}$ and were already less likely than older persons to be in secure housing, secure employment or stable partnerships. ${ }^{28}$ In contrast, older generations appear more susceptible to severe consequences of COVID-19 infection, and in many countries were recommended to 'shield' to prevent such infection. Within each generation, the pandemic's effects may have had inequitable effects by gender (eg, childcare responsibilities being borne more by women), SEP and ethnicity (eg, more likely to be in at-risk and low paid employment, insecure and crowded housing).

Using data from five nationally representative British cohort studies, which each used an identical COVID-19 follow-up questionnaire in May 2020, 
we investigated change in multiple health-impacting behaviours. Multiple outcomes were investigated since each is likely to have independent impacts on population health, and evidence-based policy decisions are likely better informed by simultaneous consideration of multiple outcomes. ${ }^{29}$ We considered multiple well-established health equity stratifiers ${ }^{30}$ : age/cohort, gender, socioeconomic position (SEP) and ethnicity. Further, since childhood SEP may impact on adult behaviours and health outcomes independently of adult SEP, ${ }^{31}$ we used previously collected prospective data in these cohorts to investigate childhood and adult SEP.

\section{METHODS}

\section{Study samples}

We used data from four British birth cohort (c) studies, born in 1946, ${ }^{32} 1958,{ }^{33} 1970^{34}$ and 2000-2002 (born 2000-2002; $2001 c$, inclusive of Northern Ireland) ${ }^{35}$; and one English longitudinal cohort study (born 1989-90; 1990c) initiated from 14 years. ${ }^{36}$ Each has been followed up at regular intervals from birth or adolescence; on health, behavioural and socioeconomic factors. In each study, participants gave written consent to be interviewed. In May 2020, during the COVID-19 pandemic, participants were invited to take part in an online questionnaire which measured demographic factors, health measures and multiple behaviours. ${ }^{37}$

\section{Outcomes}

We investigated the following behaviours: sleep (number of hours each night on average), exercise (number of days per week (ie, from 0 to 7) the participants exercised for $30 \mathrm{~min}$ or more at moderate-vigorous intensity- "working hard enough to raise your heart rate and break into a sweat") and diet (number of portions of fruit and vegetables per day (from 0 to $\geq 6$ ); portion guidance was provided). Alcohol consumption was reported in both consumption frequency (never to 4 or more times per week) and the typical number of drinks consumed when drinking (number of drinks per day); these were combined to form a total monthly consumption. For each behaviour, participants retrospectively reported levels in "the month before the coronavirus outbreak" and then during the fieldwork period (May 2020). Herein, we refer to these reference periods as before and during lockdown, respectively. In subsequent regression modelling, binary outcomes were created for all outcomes, chosen to capture high-risk groups in which there was sufficient variation across all cohort and risk factor subgroups-sleep $(1=<6$ hours or $>9$ hours per night given its non-linear relation with health outcomes), ${ }^{38} 39$ exercise ( $1=2$ or fewer days/week exercise), diet ( $1=2$ or fewer portions of fruit and vegetables/day) and alcohol $(1=\geq 14$ drinks per week or 5 or more drinks per day; $0=$ lower frequency and/or consumption). ${ }^{40}$

\section{Risk factors}

Socioeconomic position was indicated by childhood social class (at 10-14 years old), using the Registrar General's Social Class scale-I (professional), II (managerial and technical), IIIN (skilled non-manual), IIIM (skilled manual), IV (partly-skilled) and V (unskilled) occupations. Highest educational attainment was also used, categorised into four groups as follows: degree/ higher, A levels/diploma, O Levels/GCSEs or none (for 2001c we used parents' highest education as many were still undertaking education). Financial difficulties were based on whether individuals (or their parents for 2001c) reported (prior to COVID-19) as managing financially comfortably, all right, just about getting by and difficult. These ordinal indicators were converted into cohort-specific ridit scores to aid interpretation-resulting in relative or slope indices of inequality when used in regression models (ie, comparisons of the health difference comparing lowest with highest SEP). ${ }^{41}$ Ethnicity was recorded as White and non-White-with analyses limited to the 1990c and 2001c owing to a lack of ethnic diversity in older cohorts. Gender was ascertained in the baseline survey in each cohort.

\section{Statistical analyses}

We calculated average levels and distributions of each outcome before and during lockdown. Logistic regression models were used to examine how gender, ethnicity and SEP were related to each outcome, both before and during lockdown. Where the prevalence of the outcome differs across time, comparing results on the relative scale can impair comparisons of risk factor-outcome associations (eg, identical ORs can reflect different magnitudes of associations on the absolute scale). ${ }^{42}$ Thus, we estimated absolute (risk) differences in outcomes by gender, SEP and ethnicity (the margins command in Stata following logistic regression). Models examining ethnicity and SEP were gender adjusted. We conducted cohort-specific analyses and conducted meta-analyses to assess pooled associations, formally testing for heterogeneity across cohorts ( $\mathrm{I}^{2}$ statistic). To understand the changes which led to differing inequalities, we also tabulated calculated change in each outcome (decline, no change and increase) by each cohort and risk factor group. To confirm that the patterns of inequalities observed using binary outcomes was consistent with results using the entire distribution of each outcome, we additionally tabulated all outcome categories by cohort and risk factor group.

To account for possible bias due to missing data, we weighted our analysis using weights constructed from logistic regression models-the outcome was response during the COVID-19 survey, and predictors were demographic, socioeconomic, household and individual-based predictors of non-response at earlier sweeps, based on previous work in these cohorts. ${ }^{374} 44$ We also used weights to account for the stratified survey designs of the 1946c, 1990c and 2001c. Stata V.15 (StataCorp) was used to conduct all analyses. Analytical syntax to facilitate result reproduction is provided online (https://github.com/dbann/covid_ cohorts_health_beh).

\section{RESULTS}

Cohort-specific responses were as follows: 1946c: 1258 of 1843 (68\%); 1958c: 5178 of 8943 (58\%), 1970c: 4223 of 10458 (40\%); 1990c: 1907 of 9380 (20\%); 2001c: 2645 of 9946 (27\%). The following factors, measured in prior data collections, were associated with increased likelihood of response in this COVID-19 dataset: being female, higher education attainment, higher household income and more favourable self-rated health. Valid outcome data were available in both before and during lockdown periods for the following: sleep, $\mathrm{N}=14171$; exercise, $\mathrm{N}=13$ 997; alcohol, $\mathrm{N}=14297$; fruit/vegetables, $\mathrm{N}=13623$.

\section{Overall changes and cohort differences}

Outcomes before and during lockdown were each moderately highly positively correlated-Spearman's R as follows: sleep $=0.55$, exercise $=0.58$, alcohol (consumption frequency) $=0.76$ and fruit/vegetable consumption $=0.81$. For all outcomes, older cohorts were less likely to report change in behaviour compared with younger cohorts (online supplemental table 1). 
Table 1 Participant characteristics: data from 5 British cohort studies

\begin{tabular}{|c|c|c|c|c|c|}
\hline & \multicolumn{3}{|c|}{ Cohort study, birth year } & \multirow[b]{2}{*}{1958} & \multirow[b]{2}{*}{1946} \\
\hline & 2001 & 1990 & 1970 & & \\
\hline \multicolumn{6}{|l|}{ Cohort characteristics and risk factors } \\
\hline Age in years & $19-20$ & $30-31$ & 50 & 62 & 74 \\
\hline Men (\%) & 49.4 & 43.6 & 51.3 & 50.9 & 50.4 \\
\hline Father's social class, \% manual & $23.1 \%$ & $37.7 \%$ & $58.3 \%$ & $63.1 \%$ & $67.3 \%$ \\
\hline Financial difficulties, \% difficult & $18.1 \%$ & $16.1 \%$ & $21.0 \%$ & $13.4 \%$ & $4.3 \%$ \\
\hline \multicolumn{6}{|l|}{ Outcomes } \\
\hline Pre: sleep (no of hours/day), mean (SD) & $7.5(1.4)$ & $7.1(1.1)$ & $6.8(1.2)$ & $7.0(1.2)$ & $6.9(1.2)$ \\
\hline During: sleep (no of hours/day), mean (SD) & $8.1(1.9)$ & $7.4(1.5)$ & $6.9(1.5)$ & $7.0(1.4)$ & $6.9(1.3)$ \\
\hline Pre: sleep, \% atypical ( $<6>9$ hours/night) & $12.9 \%$ & $6.9 \%$ & $12.0 \%$ & $10.0 \%$ & $10.8 \%$ \\
\hline Pre: exercise (\% 0-2 days/week) & $28.8 \%$ & $32.0 \%$ & $29.7 \%$ & $26.9 \%$ & $29.5 \%$ \\
\hline During: exercise (\% 0-2 days/week) & $30.4 \%$ & $32.5 \%$ & $29.2 \%$ & $29.1 \%$ & $30.8 \%$ \\
\hline Pre: alcohol intake, $\%$ never & $15.3 \%$ & $20.5 \%$ & $16.1 \%$ & $16.2 \%$ & $18.5 \%$ \\
\hline During: alcohol intake, \% never & $27.7 \%$ & $25.2 \%$ & $19.0 \%$ & $20.9 \%$ & $20.7 \%$ \\
\hline Pre: alcohol intake, $\%$ high risk & $32.6 \%$ & $16.0 \%$ & $17.3 \%$ & $17.2 \%$ & $14.2 \%$ \\
\hline During: alcohol intake, $\%$ high risk & $13.0 \%$ & $12.7 \%$ & $21.7 \%$ & $17.4 \%$ & $14.6 \%$ \\
\hline Pre: fruit/veg intake (no of portions), mean (SD) & $3.0(1.5)$ & $3.4(1.5)$ & $3.5(1.6)$ & $3.7(1.5)$ & $4.0(1.4)$ \\
\hline During: fruit/veg intake (no of portions), mean (SD) & $3.2(1.6)$ & $3.5(1.6)$ & $3.5(1.6)$ & $3.7(1.6)$ & $4.0(1.4)$ \\
\hline Pre: fruit/veg intake (\% 0-2 portions) & $39.4 \%$ & $27.0 \%$ & $27.4 \%$ & $24.3 \%$ & $16.3 \%$ \\
\hline During: fruit/veg intake ( $\% 0-2$ portions) & $34.4 \%$ & $26.9 \%$ & $27.3 \%$ & $24.5 \%$ & $14.7 \%$ \\
\hline
\end{tabular}

Estimates are weighted to account for survey non-response. High-risk drinking is consuming more than 14 drinks a week or more than 5 drinks in a typical drinking day.

The average (mean) amount of sleep (hours per night) was either similar or slightly higher during compared with before lockdown. In each cohort, the variance was higher during lockdown (table 1) -this reflected the fact that more participants reported either reduced or increased amounts of sleep during lockdown (figure 1). In 2001c compared with older cohorts, more participants reported increased amounts of sleep during lockdown (figure 1, online supplemental tables 1 and 2). Mean exercise frequency levels were similar during and before lockdown (table 1). As with sleep levels, the variance was higher during lockdown, reflecting both reduced and increased amounts of exercise during lockdown (figure 1, online supplemental table 2). In 2001c, a larger fraction of participants reported transitions to no alcohol consumption during lockdown than in older cohorts (table 1, online supplemental table 2). Fruit and vegetable intake was broadly similar before and during lockdown, although increases in consumption were most frequent in 2001c compared with older cohorts (figure 1, online supplemental table 1).

\section{Gender inequalities}

Women had a higher risk than men of atypical sleep levels (ie, $<6$ or $>9$ hours), and such differences were larger during compared with before lockdown (pooled per cent risk difference during (men vs women, during lockdown: -4.2 ( -6.4 , $-1.9)$, before: $-1.9(-3.7,-0.2)$; figure 2$)$. These differences were similar in each cohort $\left(\mathrm{I}^{2}=0 \%\right.$ and $11.6 \%$ respectively) and reflected greater change in female sleep levels during lockdown (online supplemental table 1). Before lockdown, in all cohorts women undertook less exercise than men; during lockdown, this difference reverted to null (figure 2 ). This was due to relatively more women reporting increased exercise levels during lockdown compared with before (online supplemental table 1). Men had higher alcohol consumption than women, and reported lower fruit and vegetable intake; effect estimates were slightly weaker during compared with before lockdown (figure 2).

\section{Socioeconomic inequalities}

Those with lower education had higher risk of atypical sleep levels-this difference was larger and more consistently found across cohorts during compared with before lockdown (figure 2). Lower education was also associated with lower exercise participation, and with lower fruit and vegetable intake (particularly strongly in 2001c), but not with alcohol consumption; estimates of association were similar before and during lockdown (figure 2). Associations of childhood social class and adulthood financial difficulties with these outcomes were broadly similar to those for education attainment (online supplemental figure 1)differences in sleep during lockdown were larger than before, and lower childhood social class was more strongly related to lower exercise participation during lockdown (online supplemental figure 1), and with lower fruit and vegetable intake (particularly in 2001c).

\section{Ethnic inequalities}

Ethnic minorities had higher risk of atypical sleep levels than white participants, with larger effect sizes during compared with 


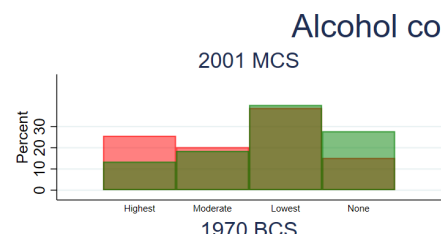

Alcohol consumption
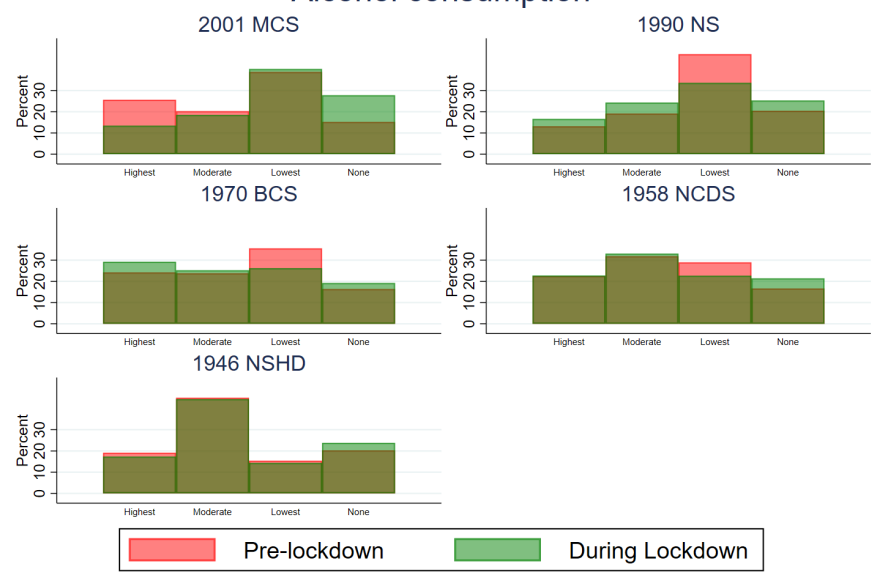

Number of days 30mins exercise 2001 MCS
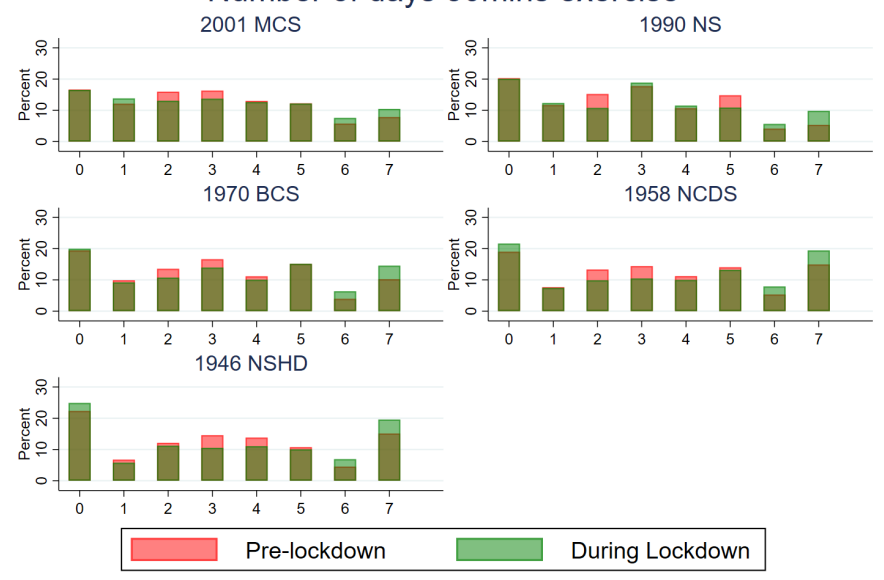
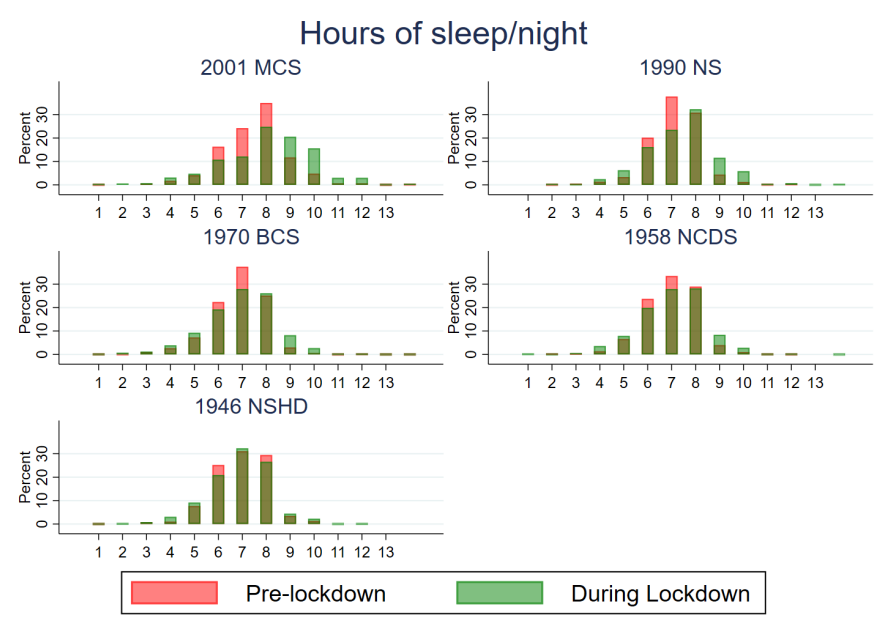

Portions of fruit and veg/day
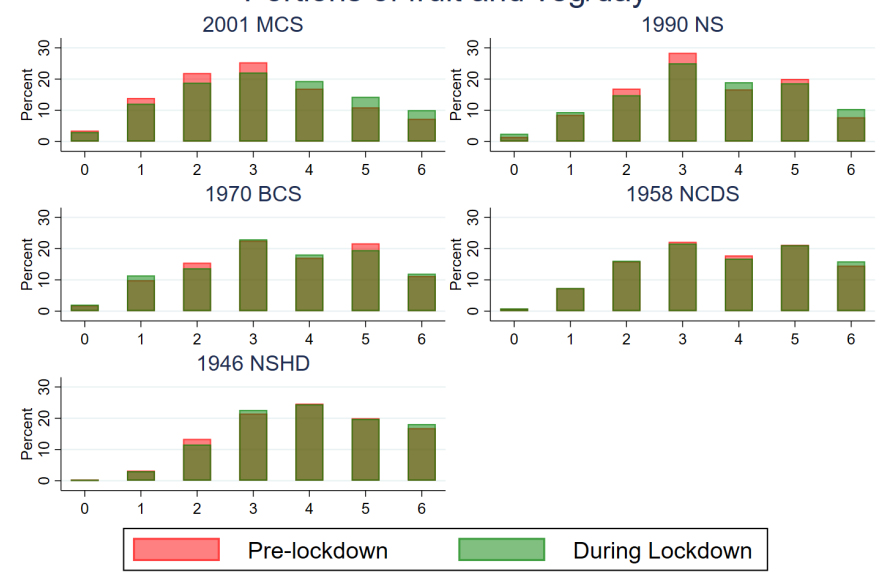

Figure 1 Before and during COVID-19 lockdown distributions of health-related behaviours, by cohort. Note: colour version of the figure is available online - Pre-lockdown = pink; During Lockdown = light green; dark green shows overlap, estimates are weighted to account for survey non-response; alcohol consumption was derived as $>36,16-36,1-15$, no drinks per month.

before lockdown (figure 2, online supplemental table 1). Ethnic minorities had lower exercise levels during but not before lockdown-pooled per cent risk difference during (ethnic minority vs white): 9.0 (1.8, $16.3 ; \mathrm{I}^{2}=0 \%$; figure 2$)$. Ethnic minorities also had higher risk of lower fruit and vegetable intake, with stronger associations during lockdown (figure 2). In contrast, ethnic minorities had lower alcohol consumption, with stronger effect sizes before lockdown than during (figure 2).

\section{DISCUSSION}

\section{Main findings}

Using data from five national British cohort studies, we estimated the change in multiple health behaviours between before and during COVID-19 lockdown periods in the UK (May 2020). Where change in these outcomes was identified, it occurred in both directions-that is, shifts from the middle part of the distribution to both declines and increases in sleep, exercise and alcohol use. In the youngest cohort (2001c), the following shifts were more evident: increases in exercise, fruit and vegetable intake, and sleep, and reduced alcohol consumption frequency. Across all outcomes, older cohorts were less likely to report changes in behaviour. Our findings suggest-for most outcomes measured-a potential widening of inequalities in healthimpacting behavioural outcomes which may have been caused by the COVID-19 lockdown.

\section{Comparison with other studies}

In our study, the youngest cohort reported increases in sleep during lockdown-similar findings of increased sleep have been reported in many ${ }^{13171824}$ but not all $^{8}$ previous studies. Both too much and too little sleep may reflect, and be predictive of, worse mental and physical health. ${ }^{38}{ }^{39}$ In this sense, the increasing dispersion in sleep we observed may reflect the negative consequences of COVID-19 and lockdown. Women, those of lower SEP and ethnic minorities were all at higher risk of atypical sleep levels. It is possible that lockdown restrictions and subsequent increases in stress-related to health, job and family concernshave affected sleep across multiple generations and potentially exacerbated such inequalities. Indeed, work using household panel data in the UK has observed marked increases in anxiety and depression in the UK during lockdown that were largest among younger adults. ${ }^{4}$

Our findings on exercise add to an existing but somewhat mixed evidence base. Some studies have reported declines in both self-reported ${ }^{1223}$ and accelerometery-assessed physical activity, ${ }^{19}$ yet this is in contrast to others which report an increase, ${ }^{22}$ and there is corroborating evidence for increases in some forms of physical activity since online searches for exercise and physical activity appear to have increased. ${ }^{21}$ As in our study, another also reported that men had lower exercise levels during lockdown. ${ }^{20}$ While we cannot be certain that our findings reflect all changes 

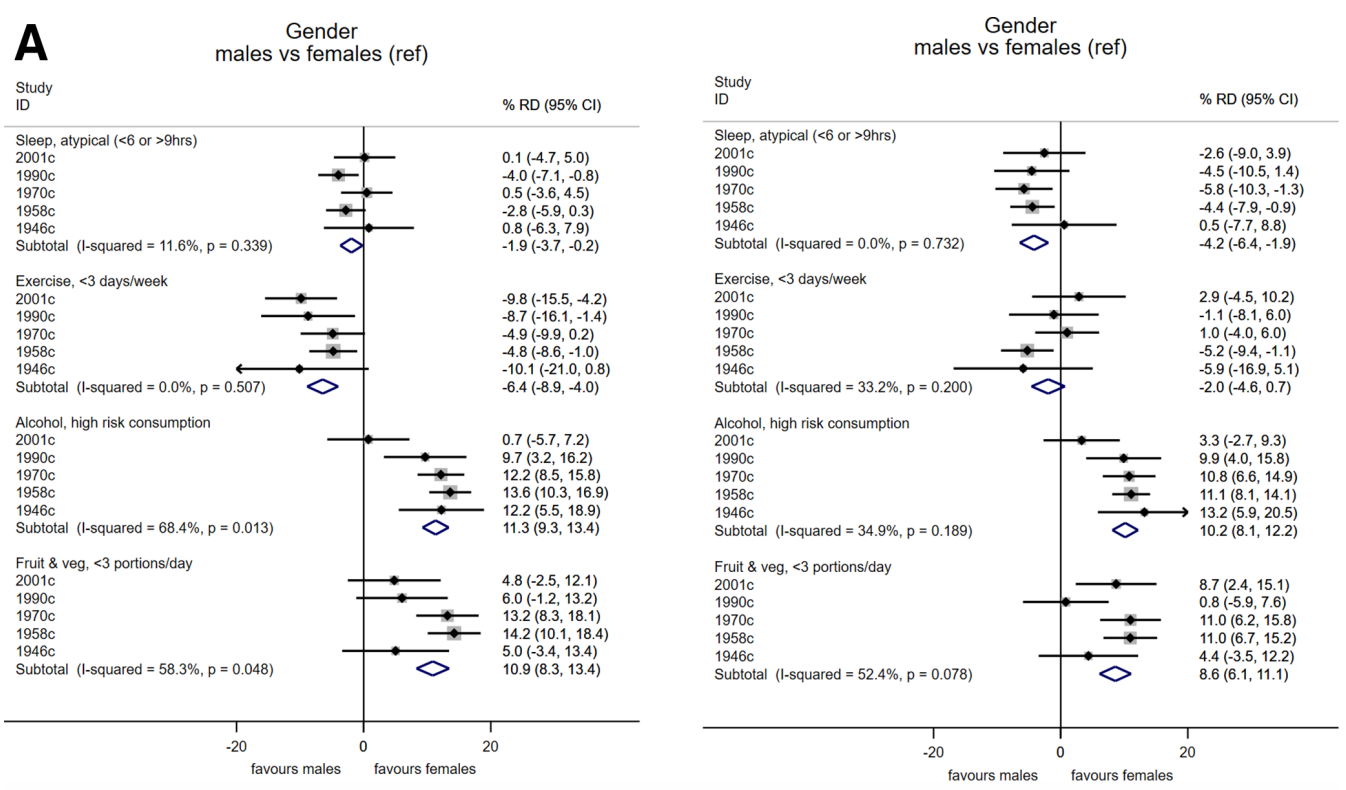

\section{B $\quad \begin{aligned} & \text { Highest education attainment } \\ & \text { lowest vs highest (ridit score) }\end{aligned}$}

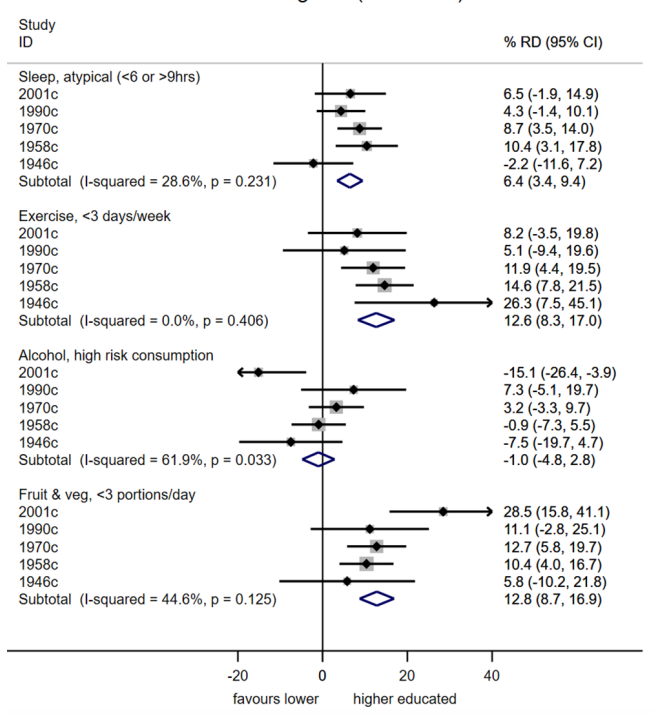

Highest education attainment lowest vs highest (ridit score)
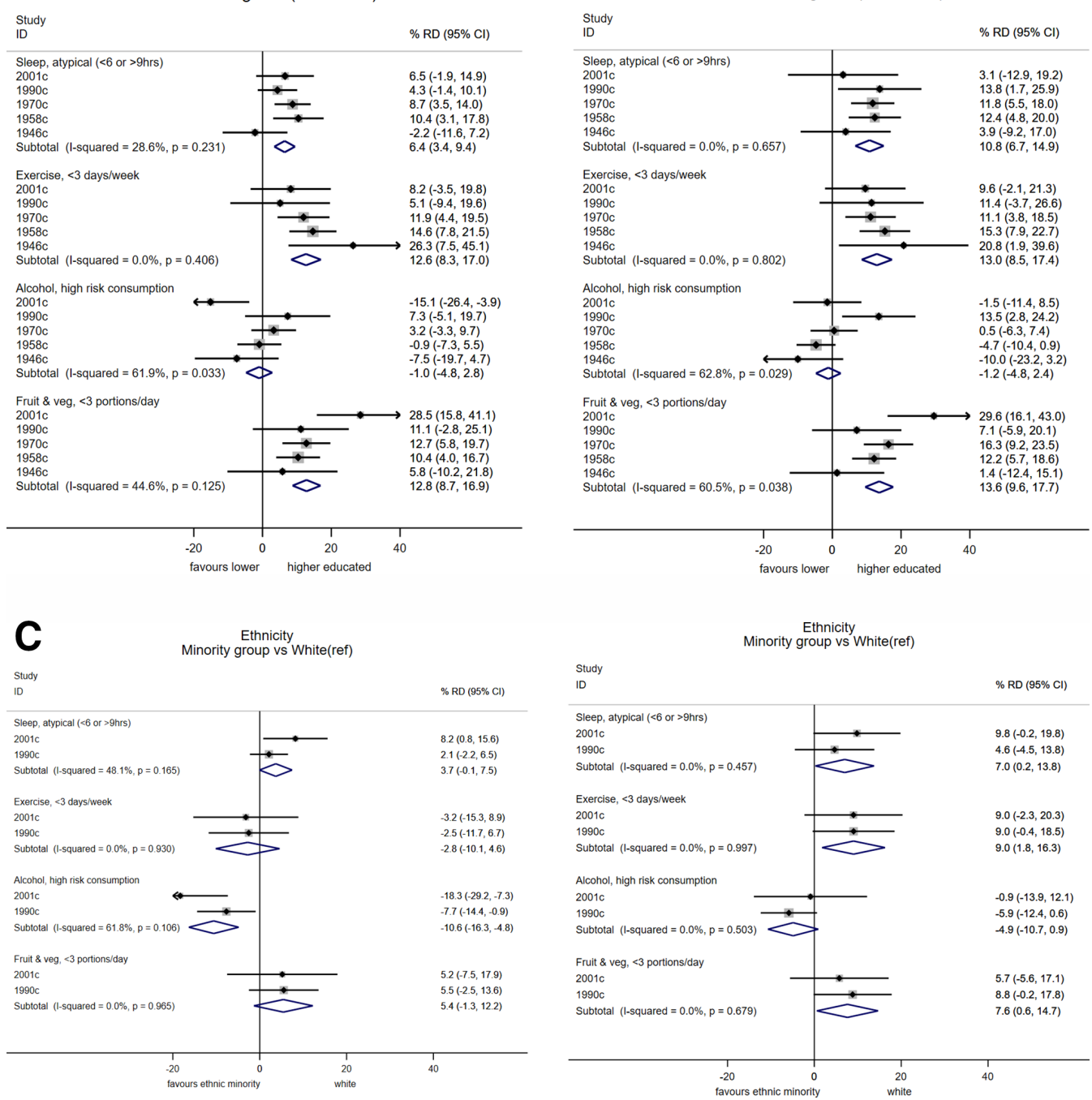

Figure 2 Differences in multiple health behaviours during COVID-19 lockdown (May 2020; right panels) compared with prior levels (left panels), according to gender (A), education attainment (B) and ethnicity (C): meta-analyses of 5 cohort studies. Note: estimates show the risk difference (RD) on the percentage scale and are weighted to account for survey non-response; ridit scores represent the difference in risk of the highest versus lowest education. 
to physical activity levels-lower intensity exercises were not assessed nor was activity in other domains such as in work or travel-the widening inequalities in ethnic minority groups may be a cause of public health concern.

As for the impact of the lockdown on alcohol consumption, concern was initially raised over the observed rises in alcohol sales in stores at the beginning of the pandemic in the $\mathrm{UK}^{45}$ and elsewhere. Our findings suggest decreasing consumption particularly in the younger cohort. Existing studies appear largely mixed, some suggesting increases in consumption, 91626 with others reporting decreases ${ }^{11} 122325$; others also report increases, yet use instruments which appear to particularly focus on capturing increases and not declines. ${ }^{810}$ Different methodological approaches and measures used may account for inconsistent findings across studies, along with differences in the country of origin and characteristics of the sample. The closing of pubs and bars and associated reductions in social drinking likely underlies our finding of declines in consumption among the youngest cohort; loss of employment and income may have also particularly affected purchasing power in younger cohorts (as suggested in the higher reports of financial difficulties (table 1)), thereby affecting consumption. Increases in fruit and vegetable consumption observed in this cohort may have also reflected the considerable social changes attributable to lockdown, including more regular food consumption at home. However, in our study only positive aspects of diet (fruit and veg consumption) were captured-we did not capture information on volume of food, snacking and consumption of unhealthy foods. Indeed, one study reported simultaneous increases in consumption of fruit and vegetables and high sugar snacks. ${ }^{11}$

Further research using additional waves of data collection is required to empirically investigate if the changes and inequalities observed in the current study persist into the future. If the changes persist and/or widen, given the relevance of these behaviours to a range of health outcomes including chronic conditions, COVID-19 infection consequences and years of healthy life lost, the public health implications of these changes may be long-lasting.

\section{Methodological considerations}

While our analyses provide estimates of change in multiple important outcomes, findings should be interpreted in the context of the limitations of this work, with fieldwork necessarily undertaken rapidly. First, self-reported measures were used-while the two reference periods for recall were relatively close in time, comparisons of change in behaviour may have been biased by measurement error and reporting biases. Further, single measures of each behaviour were used which do not fully capture the entire scope of the health-impacting nature of each behaviour. For example, exercise levels do not capture less intensive physical activities, nor sedentary behaviour; while fruit and vegetable intake is only one component of diet. As in other studies investigating changes in such outcomes, we are unable to separate out change attributable to COVID-19 lockdown from other causes-these may include seasonal differences (eg, lower physical activity levels in the pre-COVID-19 winter months), and other unobserved factors which we were unable to account for. If these factors affected the sub-groups we analysed (gender, SEP, ethnicity) equally, our analysis of risk factors of change would not be biased due to this. We acknowledge that quantifying change and examining its determinants is notoriously methodologically challenging-such considerations informed our analytical approach (eg, to avoid spurious associations, we

\section{What is already known on this subject}

- Behaviours are important contributors to population health and its equity. COVID-19 and consequent policies (eg, social distancing) are likely to have influenced such behaviours, with potential longer-term consequences to population health and its equity. However, the existing evidence base is inconsistent and challenging to interpret given likely heterogeneity across place, time and due to differences in the outcomes examined.

\section{What this study adds}

- We added to the rapidly emerging evidence base on the potential consequences of COVID-19 on multiple behavioural determinants of health. We compared multiple behaviours before and during lockdown (May 2020), across five nationally representative cohort studies of different ages (19-74 years), and examined differences across multiple health equity stratifiers: gender, socioeconomic factors across life, and ethnicity. Our findings provide new evidence on the multiple changes to behavioural outcomes linked to lockdown, and the differential impacts across generation, gender, socioeconomic circumstances across life and ethnicity. Lockdown appeared to widen some (but not all) forms of health inequality.

did not adjust for 'baseline' (pre-lockdown) measures when examining outcomes during lockdown). ${ }^{46}$

As in other web surveys, ${ }^{4}$ response rates were generally lowwhile the longitudinal nature of the cohorts enable predictors of missingness to be accounted for (via sample weights), ${ }^{43} 44$ we cannot fully exclude the possibility of unobserved predictors of missing data influencing our results. Response rates were lowest in the youngest cohorts-while the direction and magnitude of any resulting bias may be risk factor and outcome specific, unobserved contributors to missing data could feasibly bias cross-cohort comparisons undertaken. Finally, we investigated ethnicity using a binary categorisation to ensure sufficient sample sizes for comparisons-we were likely underpowered to investigate differences across the multiple diverse ethnic groups which exist. This warrants future investigation given the substantial heterogeneity within these groups and likely differences in behavioural outcomes.

\section{CONCLUSION}

Our findings highlight the multiple changes to behavioural outcomes that may have occurred due to COVID-19 lockdown, and the differential impacts-across generation, gender, socioeconomic disadvantage (in early and adult life) and ethnicity. Such changes require further monitoring given their possible implications to population health and the widening of health inequalities.

Acknowledgements We thank the Survey, Data, and Administrative teams at the Centre for Longitudinal Studies and Unit for Lifelong Health and Ageing, UCL, for enabling the rapid COVID-19 data collection to take place. We also thank Professors Rachel Cooper and Mark Hamer for helpful discussions during the COVID-19 questionnaire design period. DB is supported by the Economic and Social Research 
Council (grant no. ES/M001660/1) and Medical Research Council (MR/V002147/1); DB and AV are supported by The Academy of Medical Sciences/Wellcome Trust ("Springboard Health of the Public in 2040" award: HOP001/1025).

Contributors DB and all authors contributed to the design of the study. DB wrote the first draft and is guarantor; all authors revised this and inputted into interpretation. Conducted analyses and cleaned data: DB; audited and amended analyses: AV and JM.

Funding Medical Research Council (MR/V002147/1), Academy of Medical Sciences (HOP001/1025), Economic and Social Research Council (ES/M001660/1, ES/ V012789/1).

Competing interests None declared.

Patient consent for publication Not required.

Ethics approval Research ethics approval was obtained from the UCL Institute of Education Research Ethics Committee (ref. REC1334).

Provenance and peer review Not commissioned; externally peer reviewed.

Data availability statement Data are available from the UK Data Archive (https://beta.ukdataservice.ac.uk/datacatalogue/studies/study?id=8658\#!/https:// beta.ukdataservice.ac.uk/datacatalogue/studies/study?id=8658\#!/).

Supplemental material This content has been supplied by the author(s). It has not been vetted by BMJ Publishing Group Limited (BMJ) and may not have been peer-reviewed. Any opinions or recommendations discussed are solely those of the author(s) and are not endorsed by BMJ. BMJ disclaims all liability and responsibility arising from any reliance placed on the content. Where the content includes any translated material, BMJ does not warrant the accuracy and reliability of the translations (including but not limited to local regulations, clinical guidelines, terminology, drug names and drug dosages), and is not responsible for any error and/or omissions arising from translation and adaptation or otherwise.

Open access This is an open access article distributed in accordance with the Creative Commons Attribution 4.0 Unported (CC BY 4.0) license, which permits others to copy, redistribute, remix, transform and build upon this work for any purpose, provided the original work is properly cited, a link to the licence is given, and indication of whether changes were made. See: https://creativecommons.org/ licenses/by/4.0/.

ORCID iDs

David Bann http://orcid.org/0000-0002-6454-626X

Praveetha Patalay http://orcid.org/0000-0002-5341-3461

\section{REFERENCES}

1 Douglas M, Katikireddi SV, Taulbut M, et al. Mitigating the wider health effects of covid-19 pandemic response. BMJ 2020;369:m1557.

2 Marmot M, Allen J. COVID-19: exposing and amplifying inequalities. J Epidemiol Community Health 2020;74:681-2.

3 Joyce R, Xu X. Sector shutdowns during the coronavirus crisis: which workers are most exposed. Institute for Fiscal Studies Briefing Note BN278 2020;6.

4 Pierce $\mathrm{M}$, Hope $\mathrm{H}$, Ford T, et al. Mental health before and during the COVID-19 pandemic: a longitudinal probability sample survey of the UK population. Lancet Psychiatry 2020;7:883-92.

5 GBD 2016 Risk Factors Collaborators. Global, regional, and national comparative risk assessment of 84 behavioural, environmental and occupational, and metabolic risks or clusters of risks, 1990-2016: a systematic analysis for the global burden of disease study 2016. Lancet 2017:390:1345-422.

6 Stringhini S, Sabia S, Shipley M, et al. Association of socioeconomic position with health behaviors and mortality. JAMA 2010:303:1159-66.

7 Williamson EJ, Walker AJ, Bhaskaran K, et al. Factors associated with COVID-19related death using OpenSAFELY. Nature 2020;584:430-6.

8 The Policy Institute. Life under lockdown: coronavirus in the UK, 2020

9 Wardell J, Kempe T, Rapinda KK. Drinking to cope during the COVID-19 pandemic: the role of external and internal stress-related factors in coping motive pathways to alcohol use, solitary drinking, and alcohol problems 2020.

10 Sidor A, Rzymski P. Dietary choices and habits during COVID-19 lockdown: experience from Poland. Nutrients 2020;12:1657.

11 Scarmozzino F, Visioli F. Covid-19 and the subsequent Lockdown modified dietary habits of almost half the population in an Italian sample. Foods 2020;9:675.

12 Rodríguez-Pérez C, Molina-Montes E, Verardo V, et al. Changes in dietary behaviours during the COVID-19 outbreak confinement in the Spanish COVIDiet study. Nutrients 2020;12:1730.

13 Ong J, Lau T, Massar S. COVID-19 related mobility reduction: heterogenous effects on sleep and physical activity rhythms. arXiv preprint arXiv: 2020:200602100.
14 Mitchell ES, Yang Q, Behr H. Self-reported food choices before and during COVID-19 lockdown. medRxiv2020.

15 Marelli S, Castelnuovo A, Somma A, et al. Impact of COVID-19 lockdown on sleep quality in university students and administration staff. J Neurol 2021;268:8-15.

16 Koopmann A, Georgiadou E, Kiefer F, et al. Did the general population in Germany drink more alcohol during the COVID-19 pandemic lockdown? Alcohol Alcohol 2020;55:698-9.

17 Leone MJ, Sigman M, Golombek DA. Effects of lockdown on human sleep and chronotype during the COVID-19 pandemic. Curr Biol 2020;30:R930-1.

18 Fitbit. The impact of coronavirus on global sleep patterns, 2020. Available: https:// blog.fitbit.com/covid-19-sleep-patterns/

19 Fitbit. The impact of coronavirus on global activity, 2020. Available: https://blog.fitbit. com/covid-19-global-activity/

20 Giustino V, Parroco AM, Gennaro A, et al. Physical activity levels and related energy expenditure during COVID-19 quarantine among the Sicilian active population: a cross-sectional online survey study. Sustainability 2020;12:4356.

21 Ding D, Del Pozo Cruz B, Green MA, et al. Is the COVID-19 lockdown nudging people to be more active: a big data analysis. Br I Sports Med 2020;54:1183-4.

22 Di Renzo L, Gualtieri P, Pivari F, et al. Eating habits and lifestyle changes during COVID-19 lockdown: an Italian survey. J Trans/ Med 2020;18:1-15.

23 Deschasaux-Tanguy M, Druesne-Pecollo N, Esseddik Y. Diet and physical activity during the COVID-19 lockdown period (March-May 2020): results from the French NutriNet-Sante cohort study. medRxiv 2020.

24 Cellini N, Canale N, Mioni G, et al. Changes in sleep pattern, sense of time and digital media use during COVID-19 lockdown in Italy. J Sleep Res 2020;29:e13074.

25 Ammar A, Brach M, Trabelsi K, et al. Effects of COVID-19 home confinement on eating behaviour and physical activity: results of the ECLB-COVID19 international online survey. Nutrients 2020;12:1583.

26 Biddle N, Edwards B, Gray M. Alcohol consumption during the COVID-19 period: May 2020. COVID-19 Briefing Paper 2020

27 Singer JD, Willett JB. Applied longitudinal data analysis: modeling change and event occurrence. Oxford university press, 2003.

28 Gardiner L. Stagnation generation: the case for renewing the intergenerational contract. Resolution Foundation, 2016.

29 VanderWeele TJ. Outcome-wide epidemiology. Epidemiology 2017;28:399-402.

30 World Health Organization. Handbook on health inequality monitoring: with a special focus on low- and middle-income countries. World Health Organization, 2013.

31 Elhakeem A, Cooper R, Bann D, et al. Childhood socioeconomic position and adult leisure-time physical activity: a systematic review. Int I Behav Nutr Phys Act 2015;12:92.

32 Wadsworth M, Kuh D, Richards M, et al. Cohort profile: the 1946 National Birth Cohort (MRC National Survey of Health and Development). Int J Epidemiol 2006;35:49-54.

33 Power C, Elliott J. Cohort profile: 1958 British birth cohort (National Child Development Study). Int J Epidemiol 2006;35:34-41.

34 Elliott J, Shepherd P. Cohort profile: 1970 British birth cohort (BCS70). Int J Epidemiol 2006;35:836-43

35 Connelly R, Platt L. Cohort profile: UK Millennium Cohort Study (MCS). Int J Epidemiol 2014;43:1719-25.

36 Calderwood L, Sanchez C. Next Steps (formerly known as the Longitudinal Study of Young People in England). Open Health Data 2016;4.

37 Brown M, Goodman A, Peters A, et al. COVID-19 survey in five national longitudinal studies: wave 1 user guide (version 1). London: UCL Centre for Longitudinal Studies and MRC Unit for Lifelong Health and Ageing, 2020.

38 Jike M, Itani O, Watanabe N, et al. Long sleep duration and health outcomes: a systematic review, meta-analysis and meta-regression. Sleep Med Rev 2018:39:25-36.

39 Luyster FS, Strollo PJ, Zee PC, et al. Sleep: a health imperative. Sleep 2012;35:727-34.

40 Department of Health. UK chief medical officers' low risk drinking quidelines, 2016.

41 Mackenbach JP, Kunst AE. Measuring the magnitude of socio-economic inequalities in health: an overview of available measures illustrated with two examples from Europe. Soc Sci Med 1997;44:757-71.

42 King NB, Harper S, Young ME. Use of relative and absolute effect measures in reporting health inequalities: structured review. BMJ 2012;345:e5774.

43 Silverwood RJ, Calderwood L, Sakshaug JW, et al. A data driven approach to understanding and handling non-response in the Next Steps cohort. CLS Working Paper 2020/5. London: UCL Centre for Longitudinal Studies, 2020.

44 Mostafa T, Narayanan M, Pongiglione B, et al. Improving the plausibility of the missing at random assumption in the 1958 British birth cohort: a pragmatic data driven approach. Journal of Clinical Epidemiology 2021;136:44-54.

45 Office for National Statistics. Retail sales, Great Britain: March 2020, 2020

46 Glymour MM, Weuve J, Berkman LF, et al. When is baseline adjustment useful in analyses of change? An example with education and cognitive change. $\mathrm{Am} J$ Epidemiol 2005;162:267-78. 